\title{
The influence of Prior Knowledge on Learning Scientific Terminology: A Corpus-based Cognitive Linguistic Study of ACCELERATION in Arabic and English
}

\author{
Hicham Lahlou \\ Department of English Language Studies, School of Humanities, \\ Universiti Sains Malaysia, Penang, Malaysia \\ Hajar Abdul Rahim \\ Department of English Language Studies, School of Humanities, \\ Universiti Sains Malaysia, Penang, Malaysia
}

\begin{abstract}
The current paper expands on previous work done on the influence of learners' language and preexisting knowledge on understanding physics terminology by exploring the concept of ACCELERATION in Arabic and English. The study attempts to answer two questions: (1) what are the similarities and differences between the polysemy of Arabic تَسَارُ ع (tasāru') (acceleration) and the polysemy of English acceleration, and (2) to what extent do prototypes and factors motivating

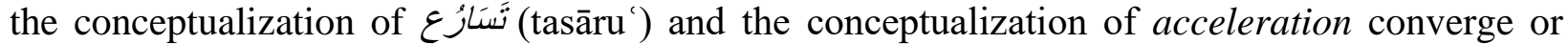
diverge? To this end, Arabic and English dictionaries and corpora, the ArabiCorpus (Arabic Corpus Search Tool) and the British National Corpus (BNC), were employed. The dictionaries were surveyed to explore the various meanings of (tasāru') and acceleration, while the ArabiCorpus and the $B N C$ were employed to investigate the senses and to identify the most frequent collocates and so the prototypes of these terms. The meaning extension of the terms is examined on the basis of the cognitive mechanisms which appear in the corpora. Theoretically, the paper is informed by the prototype theory (Rosch, 1973; 1975), image schemas (Johnson, 1987), and conceptual metaphor (Lakoff \& Johnson, 1980/2003). The results show that (tasāru') (acceleration) and acceleration generally overlap in terms of polysemy, prototype, and images schemas as well as conceptual metaphor that organize the conceptualization of these terms. It was also found that both Arab and English speakers mix ACCELERATION up with SPEED and so misunderstand them in a scientific setting. The present findings have several implications for science curriculum design, education, and research on universal and culture-specific properties of language.
\end{abstract}

Keywords: acceleration, conceptual metaphor, image schemas, polysemy, prototype

Cite as: Hicham Lahlou, H., \& Rahim, H. A. (2020). The influence of Prior Knowledge on Learning Scientific Terminology: A Corpus-based Cognitive Linguistic Study of ACCELERATION in Arabic and English. Arab World English Journal for Translation \& Literary Studies 4 (1) 148-160.

DOI: http://dx.doi.org/10.24093/awejtls/vol4no1.12 